\title{
A study of the near-miss involving Weber's law and pure-tone intensity discrimination'
}

\author{
W. J. McGILL ${ }^{2}$ AND J. P. GOLDBERG \\ DEPARTMENT OF PSYCHOLOGY, UNIVERSITY OF CALIFORNIA, SAN DIEGO
}

A study of pure-tone intensity discrimination is presented in which amplitude changes are detected in $1000 \mathrm{~Hz}$ tone bursts $15-20$ msec in duration. The masking function log detectable increment vs log background intensity') is found to have a slope of 9/10 when calculations are carried out via energy measurements. This near-miss to Weber's law is in agreement with other data reported in the literature. The masking slope proves to be essentially independent of stimulus duration between 15 msec and 1.5 sec. Our stable slope parameter is interpreted as a detectability restriction generated by "mass-flow" phenomena in the alditory channel. These phenomena are thought to be similar to the fluctuations accompanying a noisy or turbulent stream of events. Pure-tone intensity discrimination is then analyzed as a special case of energy detection.

Two pure tones at the same frequency must exhibit a certain minimum intensity difference in order to be detectably different to the ear. The intensity difference may then be expressed in ratio with a baseline provided by either of the two bounding tones, to form a Weber-fraction. Weber-fractions determined in this way at successively higher intensities, outline a curve described here as a "Weber-function." When the curve is flat, the function is said to be following Weber's law, as every student knows. Classical data show the Weber-function for pure tones as falling off, rapidly at first then slowly, with increasing intensity (Riesz, 1928). This observation is often summoned to service in textbooks in order to show what every student is also expected to know; i.e., that Weber's law fails for pure tones (see, for example, Woodworth \& Schlosberg, 1954, pp. 222-225).

Recently, pure-tone intensity discrimination has attracted renewed attention. It has been discovered that while Weber's law does not hold in the strict sense, it misses connections in a most interesting way. The masking function (log detectable increment vs log baseline intensity) is found to be remarkably linear above 25 $\mathrm{dB}$ sensation level. The slope of this linear function is not, however, unity as Weber's law would decree. Instead, the pure-tone masking slope measures at approximately $9 / 10$. This slope figure is observed whenever increment energy is added coherently to background energy using $1000 \mathrm{~Hz}$ pure tones (Dimmick \& Olson, 1941; Campbell \& Lasky, 1967; Green, 1967: McGill \& Goldberg, 1968). Slope does not seem to change with frequency, but exploration is still incomplete.

The near-miss to Weber's law must be deemed important because an analogous slope parameter computed for sinusoids detected in wideband noise, turns out to be almost exactly unity (Hawkins \& Stevens, 1950). Moreover, unit slope also characterizes intensity discrimination in pure noise (Miller, 1947; Raab et al, 1963). Why then should the masking function for pure tones display a characteristic slope depression in comparison with noise: The question is essentially unanswered.

This paper presents a new pure-tone masking curve. Our tone frequency is $1000 \mathrm{~Hz}$ and our stimulus durations are 15 and 20 msec. The slope of the masking function is found to be $9 / 10$ in good agreement with other data. These results are first compared with an earlier study of the masking slope obtained with $1000 \mathrm{~Hz}$ stimuli $150 \mathrm{nsec}$ in duration (McGill \& Goldberg, 1968). Similar comparisons are then developed for masking data in the literature. We show that at $1000 \mathrm{~Hz}$ the masking slope is approximately $9 / 10$ and is essentially independent of stimulus duration from $15 \mathrm{msec}$ to $1.5 \mathrm{sec}$.

What might such a stable slope parameter signify? It is interpreted here as suggesting mass-flow phenomenon in the auditory system. similar in character to loudness. We seek to build a bridge between pure tones and noise by applying the idea of mass information-flow to both in order to generate an explanation of masking phenomena. "Mass information-flow" is a term used here to describe a communication system having major information losses. The latter are attributable to collapsing or smearing a volume of information-bearing elements together, and dealing with them en masse instead of in detail. We show that this approach can predict both the unit slope observed with noise, and the slightly depressed slope characteristic of pure tones.

It is perhaps proper to speak of intensity discrimination instead of masking in describing our data. The data are based on loud-soft judgments of single, gated tones. There is no need, however, to be too fastidious about the distinction. The functions we study turn out to be very similar to masking functions. Single tone intensity discrimination and masking are evidently closely related.

\section{PROCEDURE}

The experimental format is a Yes-No detection task with feedback. A sine wave oscillator (HP-200CD), set for $1000 \mathrm{~Hz}$, was gated on at the positive-going zero crossing of the waveform. The gate remained open for 15 or $20 \mathrm{msec}$ as determined by a preset counter and interval timer. Rise and decay times were fixed at 2.5 msec. This single, gated pure-tone was then switched by a probability programmer (BRS-PP1) into one of two attenuators set for intensity differences ranging from $0 \mathrm{~dB}$ to $5.0 \mathrm{~dB}$ in $.1 \mathrm{~dB}$ steps. The output of the live attenuator was patched into a soundproof chamber to a single Telefonix TDH-39 earphone mounted in a MX/41-AR cushion.

Experimental sessions consisted of sequences of such tones. Each tone was at one of two intensities and intensities were presented in random order. A green warning light terminated with the onset of the stimulus tone. S pushed his response key to the left or right according as the tone he heard seemed to be the softer or the louder member of the pair. A red feedback light then flashed on at the left or right of a panel immediately in front of $S$ indicating which stimulus was actually presented. This concluded a trial and the program recycled, repeating itself every $8.4 \mathrm{sec}$. The trial sequence was as follows:

$\begin{array}{ll}\text { Warning } & (700 \mathrm{msec}) \\ \text { Tone } & (20 \mathrm{msec}) \\ \text { Response Interval } & (2.1 \mathrm{sec}) \\ \text { Feedback } & (2.1 \mathrm{sec}) \\ \text { Intertrial Interval } & (3.5 \mathrm{sec})\end{array}$

Timing was established by Tektronix 160 series pulse and waveform generators. Switching and recording were controlled by a Digibit network. Stimuli and responses were printed out on adding machine tape.

Listeners were three college students (two male; one female). They were given from 4 to $25 \mathrm{~h}$ of preliminary training in the detection task. Ss were run in 2-h test sessions. Approximately 20 such sessions were recjuired in order to generate psychometric functions at nine different intensities. Blocks of 70-80 trials were run with brief rest intervals between blocks. Points on the psychometric functions are computed from some 150-250 measurements.

The more intense member of each pair of alternatives was kept constant and the intensity of the weaker stimulus was changed in order to measure the discriminability of a given decibel difference. The stronger alternative thus forms our reference level. We study what may be called "just-detectable decrements," although symmetry in the task makes an increment and a decrement essentially equivalent. When the decibel difference between alternatives was sufficiently large, they could be discriminated 
with perfect accuracy (provided they were audible). As the intensity difference decreased, the percentage of correct responses dropped to chance performance ( 50 percent correct).

\section{RESULTS}

Psychometric functions governing pure-tone intensity discrimination were obtained at various reference levels between $0 \mathrm{~dB}$ and $70 \mathrm{~dB}$ sensation level. The stimuli were $1000 \mathrm{~Hz}$ tones, phase locked, and either 15 or $20 \mathrm{msec}$ in duration. Our functions trace the percentage of correct discriminations corresponding to increasing intensity differences between two alternatives having the same frequency and duration but differing slightly in loudness. These psychometric functions resemble curves obtained in an earlier study of tones $150 \mathrm{msec}$ in duration (McGill \& Goldberg, 1968). Their most salient feature is an evident failure to produce the same Weber-fraction at different reference levels. Thus Weber's law fails. The functions are found to be roughly parallel but displaced from one another (see Fig. 1). In this respect they are quite different from psychometric functions seen in noise masking. The latter nearly superimpose. The pure-tone functions display a strong tendency to shift toward increasing sensitivity as reference level increases.

Taken together, such considerations imply that our pure-tone masking function (or intensity discrimination curve) must exhibit less than unit slope as suggested earlier. Figure 2 shows this to be the case. The upper limb of the function, extending from $20 \mathrm{~dB}$ sensation level to at least $70 \mathrm{~dB}$ sensation level, is quite linear. Its slope calculates out at .905 via least squares, and there are no serious differences among the three Ss. Unit slope is shown for comparison. Campbell and Lasky (1967) have published Weber functions at the same frequency and stimulus duration $(1000 \mathrm{~Hz}$; $20 \mathrm{msec}$ ). Their data (recomputed) show very nearly the same slope as our own. They also suggest that linearity extends at least $10 \mathrm{~dB}$ beyond our observations at $70 \mathrm{~dB}$ sensation level.

An intensity discrimination curve such as the one illustrated in Fig. 2 may be computed very simply. We first measure the observed threshold decibel difference at a given reference level. This is the point at which the psychometric function crosses $75 \%$ correct in Fig. 1. For example, when measurements were made at a reference point of $33 \mathrm{~dB}$ sensation level, the just detectable decrement was found to be $3.57 \mathrm{~dB}$, using our procedure. Label the energy level at $33 \mathrm{~dB}$ as $\mathrm{E}_{\mathrm{o}}$. The energy corresponding to 3.57 $d B$ below $33 \mathrm{~dB}$ is then indicated as $E_{o}-E_{s}$. We have for the decibel difference:

$$
10 \log \left(\frac{E_{o}}{E_{o}-E_{s}}\right)=3.57
$$

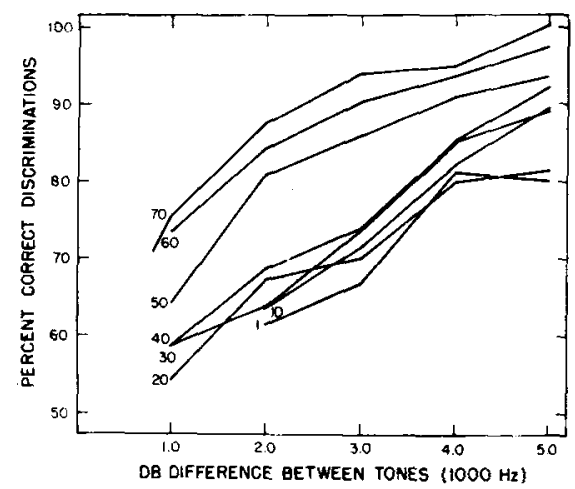

Fig. 1. Psychometric functions for pure-tone intensity discrimination. Tone are phase-locked $1000 \mathrm{~Hz}$ sinusoids, $15 \mathrm{msec}$ in duration. Two intensities are presented in random onder. One intensity is reference sensation level shown as the curve parameter. The other is attenuated by an amount shown on abscissa. Listener judges which of the two altematives (soft-loud) he hears and feedback tells him which was actually presented. Psychometric functions depict changing discriminability as $\mathrm{dB}$ difference increases. Data of one listener.

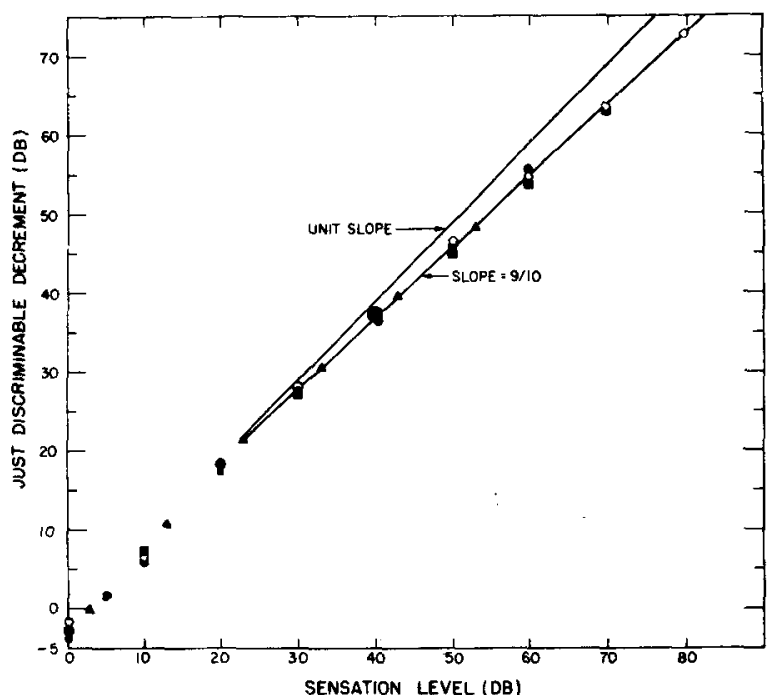

Fig. 2. Pure tone intensity discrimination curve. Abscissa is reference level of test pure tone $(1000 \mathrm{~Hz})$. Ordinate is sensation level of energy decrement just detectably different from test tone as determined by $75 \%$ discriminability points in Fig. 1 and related data. Unit slope depicts Weber's law. Actual slope fitted by least squares is $\mathbf{9 0 5}$. Data of three listeners shown [dark squares (15 msec), dark circles (15 msec), dark triangles (20 msec)]. Open circles are 20 msec gated masker data of Campbell and Laky (1967, Fig. 1). The Campbell-Lasky data are adjusted for best match to current data.

A Weber-fraction is then computed from:

$$
\frac{E_{s}}{E_{o}-E_{s}}=\frac{E_{o}}{E_{o}-E_{s}}-1,
$$

Now a routine computation can be set up using the Weber fraction, the decibel difference, and the reference level:

$$
\begin{aligned}
10 \log E_{s} & =10 \log \left(\frac{E_{s}}{E_{o}-E_{s}}\right)-10 \log \left(\frac{E_{o}}{E_{o}-E_{s}}\right)+10 \log E_{o}, \\
& =1.06 \\
& =30.49 \mathrm{~dB} .
\end{aligned}
$$

The outcome is plotted in Fig. 2 as the just detectable decrement corresponding to $33 \mathrm{~dB}$ sensation level.

An important difference exists between slope calculations obtained from energy measurements and amplitude measurements. The latter generate a slope of approximately .85 instead of the figure of .90 governing the data reported in Fig. 2. This discrepancy occurs because our sine wave decrements were subtracted in phase. It is easy to show that points on the ordinate (Fig. 2) obtained via energy computations differ from analogous computations based on amplitude measurements by a factor amounting to:

$$
10 \log \left(1+2\left[\frac{P_{o}-P_{s}}{P_{s}}\right]\right)
$$

The term in square brackets is the reciprocal of the Weber-fraction when the latter is formulated as an amplitude ratio. Since our psychometric functions fail to superimpose at different reference levels, this correction factor cannot be constant at different reference levels. Hence the slope of pure-tone intensity discrimination will depend on whether the data are construed to be amplitude measurements or energy measurements.

Weber fractions obtained via our methods seem to be fairly large. The threshold difference of $3.57 \mathrm{~dB}$ cited in earlier calculations is equivalent to $E_{s} / E_{o}=.56$ at $33 \mathrm{~dB}$ sensation level. This, of course, would not be the most sensitive measurement we have. At $60-70 \mathrm{~dB}$ sensation level $\mathrm{E}_{\mathrm{s}} / \mathrm{E}_{\mathrm{o}}$ is found to be 


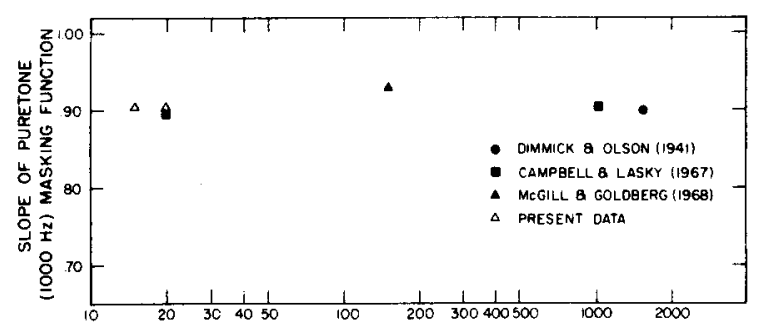

STIMULUS DURATION (MILLISECONDS)

Fig. 3. Slopes of pure tone $(1000 \mathrm{~Hz})$ energy discrimination curves plotted as a function of stimulus duration. Data in this paper shown as open triangles. Campbell and Lasky (1967) data recomputed from Weber functions. Masking slope is seen to be essentially independent of duration between $15 \mathrm{msec}$ and $1.5 \mathrm{sec}$.

approximately .20; still quite large. The magnitude of the Weber-fraction is evidently related to the single stimulus technique used here. Our method cannot be considered to be very sensitive. The size of the Weber ratio appears, however, to have little effect on the shape of the intensity discrimination curve. Figure 2 shows discrimination data obtained by Campbell and Lasky (1967) using the method of forced choice with pure tone stimuli identical to those studied here. The shape of the function is practically indistinguishable from ours.

These results suggest that the slope of pure tone intensity discrimination (beyond $20 \mathrm{~dB}$ sensation level) must be relatively impervious to two of the most prominent frustrations encountered in psychophysical analysis: method variance, and individual differences. Hence we have felt it possible to go to the literature on intensity discrimination in order to compute other slopes. Data are taken from a number of different experiments and we have used them to study the relation governing the slope of $1000 \mathrm{~Hz}$ intensity discrimination and stimulus duration. Figure 3 displays the outcome for durations from $15 \mathrm{msec}$ to $1.5 \mathrm{sec}$. It is apparent that no important slope changes occur in this range.

Dimmick and Olson (1941) offer power measurements analogous to those in Fig. 2. The slope figure of .90 reported in Fig. 3 is as given by the original authors. This excellent early paper does not seem to have received the attention it merits. Data observed by Campbell and Lasky (1967) at $20 \mathrm{msec}$ and $1000 \mathrm{msec}$ were recomputed from the original Weber functions using a table provided by D. Ronkin (personal communication).

It should be stressed that the linear functions under discussion here may be said to characterize pure-tone intensity discrimination only above $20-25 \mathrm{~dB}$ sensation level. The region below $20 \mathrm{~dB}$ in Fig. 2 exhibits a more steeply rising curve which might also be linear but with a slope very near unity. On the other hand, Campbell and Lasky (1967) describe certain of their Weber functions as having the appearance of an "inverted N." These abrupt changes in sensitivity are largely confined to the intensity region below 20-25 dB sensation level. Their sharpness is somewhat softened by an intensity discrimination plot, but we have also seen the inverted- $\mathrm{N}$ appearance with pure tones $150 \mathrm{msec}$ in duration. There may then be a systematic relation between stimulus duration and the shape of the intensity discrimination curve in the region below $20-25 \mathrm{~dB}$ sensation level. The explanation of this effect, if it is real, is not abundantly clear.

\section{DISCUSSION}

Discussion here is restricted to pure tone intensity discrimination (Fig. 2) extending some $60 \mathrm{~dB}$ above $20 \mathrm{~dB}$ sensation level. As we have observed, the data in this region may be characterized as a linear function having a slope of approximately $9 / 10$.

In an earlier paper (McGill \& Goldberg, 1968) attention was drawn to the argument that a statistical process akin to spontancous energy fluctuations can produce a linear pure-tone intensity discrimination curve. The observed slope then reflects a simple relation hetween information flow in the auditory channel and energy input to the ear. The same statistical process driven by a wideband noise generates unit slope for the intensity discrimination curve. We now propose to develop this argument.

How can energy fluctuation phenomena arise when pure tone stimuli are phase-locked and controlled in duration? This is a problem that has always frustrated applications of detection theory to pure tone masking. Two sine waves, fixed in frequency and phase, and differing slightly in amplitude, are unambiguously different. Accordingly, they should be discriminably different. Tanner (1961) sought to reintroduce stimulus ambiguity by suggesting that spontaneous fluctuations in sine-wave oscillators would be proportional to the output gain setting, thus producing masking effects similar to those observed with noise. Efforts to measure such fluctuations have generally led to the conclusion that they are much too small to account for the amount of pure tone masking typically observed (see, for example, D. M. Green, 1967 , p. 1518). Moreover, output fluctuations seem to predict unit slope for the pure-tone masking function, and this is not what we see.

It is perhaps more useful to locate the noisy process accounting for confusions between pure tones, somewhere inside the observer. The difficulty in that instance is evident from Fig. 2. Observer noise somehow manages to match itself to the stimulus intensity. They are in fact linearly related. How then can the noise be inside the observer?

There is an easy way out of this dilemma. We need only assume that internal noise arises directly out of the detection process. It is at this point that an understanding of the losses associated with mass-flow of information can help. Mass-flow phenomena are an important source of noise. In our problem the "noise" measures a characteristic loss of information associated with ignoring certain details of the stimulus representation in the auditory channel.

Suppose the effects of the gated pure tone are spread over a number of independent neural pathways due to relatively poor analytical action in the cochlea, and suppose this activity is monitored in undifferentiated form as suggested by the concept of critical bands. The effect is equivalent to superimposing the information channels within the monitored band. This in turn generates spontaneous fluctuations in short segments of the mass-flow. More particularly, when independent point-like renewal processes are superposed, and labels identifying the individual sequences are removed, brief observations of the total number of events tend toward a Poisson limiting distribution (Cox \& Smith, 1953; Drenick, 1960; Ten Hoopen, 1967).

The phenomenon is similar to the normal limit which occurs when random magnitudes are added, except that here random (or even nonrandom) intervals are interlaced. Intuitively, the Poisson limit occurs when a mechanism counting impulses from many different input channels becomes uniformly uncertain as to which input channel will generate the next impulse. Thus even though each channel may deliever a nonrandom impulse sequence, short observation periods, together with an inability to keep elaborate records, will produce the uniform uncertainty which we require. This uniform uncertainty is the hallmark of a Poisson process. A detection process that smears information together and counts it en masse, will generate an internal noise via concurrent loss of information. Moreover, the level of this noise will be tied to the Poisson mean, i.e., the rate of flow of events in the channel. This rate is determined by stimulus intensity. The detection process then will have an internal noise that increases with stimulus intensity.

This is only one of a variety of mechanisms that might be called upon to explain pure-tone masking. It is a particularly simple one, however, and it allows us to move smoothly back and forth between pure tone masking and noise masking. Tonal analysis plays no critical role in the assumed operation of the mechanism governing intensity discrimination.

We now consider how the observed pure-tone masking effects might arise. Assume that the orderly information flow back from the cochlea is focussed and counted 'n masse'. introducing fluctuations typical of energy detection. Reference intensity generates a Poisson distribution of counts in repeated observation 
intervals. The mean value of this number is labeled $n_{0}$. Similarly, when an increment (or decrement) is added to the energy of the reference signal, the Poisson mean shifts to $n_{s}+n_{o}$ where $n_{s}$ is the count attributable to the energy increment.

A device such as this obeys a characteristic detection law:

$$
\frac{\mathrm{n}_{\mathrm{s}}}{\mathrm{n}_{\mathrm{o}}{ }^{1 / 2}} \cong \mathrm{k} \text {, }
$$

where $\mathrm{k}$ is fixed when detectability is fixed. The law prescribes a square root form for intensity discrimination in the channel. It was originally presented by de Vries (1943) as a rationale for visual intensity discrimination, and is easily proved (see Appendix). We do not find a square root law (see Fig. 2) with pure tones. The curve is linear but its slope is $9 / 10$ instead of $1 / 2$. This means that if the model is presumed to be basically satisfactory, the count in the channel cannot be proportional to input energy. Proportional counting would also have to be rejected on superficial arguments. The range of audible energy spans some $13 \mathrm{log}$ units, whereas neural firing is compressed into approximately $3 \mathrm{log}$ units. There is thus no easy way in which proportionality can be established.

Let us then take the following to be our assumed energy relation:

$$
n_{s}+n_{o}=a\left(E_{s}+E_{o}\right)^{1-c}, 0 \leqslant c<1 .
$$

The parameters $a$ and $c$ reflect the operation of the sensory process in matching the counting rate to the energy input. When the parameter $\mathrm{c}$ is set to zero, we have proportional counting as before. When $c=1$, there is perfect AVC action. The sensory channel somehow compensates for the input energy level and the counting rate is essentially the same for each stimulus intensity. These boundary conditions allow us to estimate the exponent in Equation 2 from the slope of the intensity discrimination curve. The Appendix shows that Equation 2 inserted into the Poisson detection process produces as the observed discrimination law:

$$
\frac{2 a^{1 / 2}(1-b) E_{s}}{\left(1+E_{s} / E_{o}\right)^{1 / 2} E_{o} b} \cong k \text {, }
$$

where $E_{s}$ and $E_{o}$ are increment and background energy respectively, and

$$
b=\frac{1+c}{2}, 1 / 2 \leqslant b<1 .
$$

The square root term in the denominator of Equation 3 is generally small and changes slowly in the linear region of pure tone intensity discrimination. Hence we may approximate Equation 3 as follows:

$$
\log E_{s} \cong K+b \log E_{o}
$$

where $K$ absorbs all constants. Equation 4 furnishes a suitable approximation of the law governing pure tone intensity discrimination if the latter is taken to be an information-loss phenomenon in the channel, and Equation 2 is taken to be the expression governing growth of the mass-flow of information as stimulus intensity increases.

We have now managed to generalize Weber's law in a manner reminiscent of Guilford's nth power law (1936, pp. 138-139). Guilford, of course, worked on the problem long before ideas on information-flow were as well established as they are now.

Our estimated slope of $9 / 10$ acquires an important significance in this picture of intensity discrimination. It depicts a specific form for the relation between the count in the auditory channel and the in put energy level, namely:

$$
\mathrm{n}=\mathrm{aE}, 20
$$

Thirteen log units of pure-tone stimulus intensity would be compressed into $2.6 \mathrm{log}$ units of counting. The ratio between maximum and minimum counts would then be about $400 / 1$. Thus it is possible to interpret the outcome in Equation 5 as being a primitive loudness function. The form of Equation 2 was chosen with this possibility in mind, but the estimated slope was in no way predetermined. As it works out, the estimated slope proves to be somewhat smaller than the cube root law typically seen with judgments of loudness, but it is not widely at variance with the latter. We may then have found a way of relating two phenomena (loudness and intensity discrimination) which superficial analysis suggests should be related, but for which no vehicle of mutual translation has existed previously. .

The arguments used to construct the intensity discrimination law in Equation 3 can also be made to produce a simple expression for the psychometric function. In our Appendix we show that a Poisson detection process in conjunction with the energy relation in Equation 2 generates a detection formula having approximately the following form:

$$
2(1-b) n_{o}^{1 / 2} \log _{e}\left[1+\frac{E_{s}}{E_{o}}\right] \cong d^{\prime},
$$

where $d^{\prime}$ is the familiar standard normal measure of detectability. Equation 6 tells us that the psychometric function should increase as a cumulative normal variable corresponding to the decibel difference between pure tone alternatives. Moreover, if $d^{\prime}$ is fixed and $n_{o}$ increases (it must increase when the reference level increases), the threshold decibel difference must then decrease in order to counter this change. It follows that the psychometric functions will shift toward increasing sensitivity as reference level increases, and this is in fact what we observe.

We should note that our arguments restrict the slope of pure tone intensity discrimination between lower and upper bounds of $1 / 2$ and unity respectively. Unit slope is seen only in the unlikely circumstance of nearly perfect AVC action. The lower bound (slope $=1 / 2$ ) is attained in the equally unlikely case of proportional counting. Our investigation of duration effects was undertaken with the hypothesis in mind that the burst of neural activity following a gated pure tone, might change its overall rate characteristics as stimulus duration lengthened. This change might then be reflected as a change in the slope parameter governing intensity discrimination if our basic ideas proved to be sound. It does not seem to happen, at least with $1000 \mathrm{~Hz}$ stimuli between $15 \mathrm{msec}$ and $1.5 \mathrm{sec}$.

Suppose we apply the line of reasoning we have used in analyzing pure-tone intensity discrimination, and attempt now to analyze bursts of pure noise. The stimulus energy may no longer be treated as fixed from trial to trial. It has a probability distribution closely related to chi-square (see Green, 1960). This random variation of the stimulus is then imposed on the mean of the Poisson counting distribution with extremely interesting results. McGill (1967, p. 362) shows that proportional counting of a pure noise stimulus transforms the Poisson distribution into a negative binomial. The variance of the latter contains a Poisson variance component as well as a component due to the energy distribution of the noise stimulus. On the basis of this expanded variance, McGill (p. 364-5) establishes that the negative binomial generates an intensity discrimination curve having unit slope. Equation 5 with an exponent of the order of .20 is a slowly changing function, the proportional counting assumption is then approximately true over the range of stimulus fluctuations in the energy distribution associated with a given noise level. The proportionality "constant" changes, of course, in different parts of the energy range, but since the constant cancels out of the detection formula (McGill, 1967, Equation 32, p. 368), the slope of the intensity discrimination law is found to be unity. In pure noise, the unit slope of intensity discrimination indicates that the variance of the counting distribution is much larger than is the case with pure tones. Proportionality with the input noise level is thereby established. Accordingly, an energy detection process explains both the unit slope of noise masking and the slightly depressed slope observed with pure tones. The changing variance of the counting distribution accounts for the difference.

\section{REFERENCES}

CAMPBELL, R. A., \& LASKY, E. Z. Masker level and sinusoidal-signal detection. J. Acoust. Soc. Amer., 1967, 42, 972-976.

COX, D. R., \& SMITH, W. L. The superposition of several strictly periodic 
sequences of events. Biometrika, 1953, 40, 1-11.

DIMMICK, F. L., \& OLSON, R. M. The intensive difference limen in audition. J. Acoust. Soc. Amer., 1941, 12, 517-525.

DRENICK, R. F. The failure law of complex equipment. J. Soc. industr. appl. Math., 1960, 8, 680-690.

GREEN, D. M. Auditory detection of a noise signal. J. Acoust. Soc. Amer., $1960,32,121-131$.

GREEN, D. M. Additivity of masking. J. Acoust. Soc. Amer, 1967, 41, 1517-1525.

GUILFORD, J. P. Psychometric methods. New York: McGraw Hill, 1936.

HAWKINS, J., \& STEVENS, S. S. The masking of pure tones and speech by white noise. J. Acoust. Soc Amer., 1950, 22, 6-13.

McGILL, W. J. Poisson counting and detection in sensory systems. Unpublished manuscript, Department of Psychology, University of California, San Diego, Feb. 10, 1966. Written for Conceptual bases and applications of the information sciences, E. F. Beckenbach (Ed.); in preparation.

McGILL, W. J. Neural counting mechanisms and energy detection in audition. J. math. Psychol, 1967, 4, 351-376.

McGILL, W. J., \& GOLDBERG, J. P. Pure tone intensity discrimination and energy detection. J. Acoust. Soc. Amer., in press.

MILLER, G. A. Sensitivity to changes in the intensity of white noise and its relation to masking and loudness. J. Acoust. Soc. Amer., 1947, 19 , 609-619.

RAAB, D., OSMAN, E., \& RICH, E. Effects of waveform correlation and signal duration on detection of noise bursts in continuous noise. J. Acoust. Soc. Amer., 1963, 35, 1942-1946.

RAO, C. R. Advanced statistical methods in biometric research. New York: Wiley, 1952.

RIESZ, R. R. Differential sensitivity of the ear for pure tones. Phys. Rev., 1928, 31, 867-875.

TANNER, W. P. Application of the theory of signal detectability to amplitude discrimination. J. Acoust. Soc. Amer., 1961, 33, 1233-1 244.

TEN HOOPEN, M. Pooling of impulse sequences with application to neuronal spike data. Kybemetik, 1967, 4, 1-10.

de VRIES, H. L. The quantum character of light and its bearing upon the threshold of vision, the differential sensitivity, and acuity of the eye. Physica, 1943, 10, 553-564.

WOODWORTH, R. S., \& SCHLOSBERG, H. Experimental psychology. (rev. Ed.), New York: Holt \& Co., 1954.

\section{NOTES}

1. This research was supported by the National Science Foundation under Grant GB-5281.

2. Address: Department of Psychology, University of California, San Diego, La Jolla, Calif. 92037.

\section{(Accepted for publication April 16, 1968.)}

\section{APPENDIX}

Consider two Poisson distributions, one with mean value $\mathrm{n}_{o}$, and the second with mean value $n_{s}+n_{o}$. In order to discriminate between them a critical number $c$ is chosen. An observation is assigned to one or the other of the two possible source distributions according as the observation is or is not less than the critical number. If the latter is chosen sufficiently large, the chance that it is exceeded by observations from the background distribution, can be made arbitrarily small. In that circumstance $\mathbf{n}_{\mathrm{s}}$ must be made fairly large before there is appreciable probability that an observation from the incremental distribution will exceed the critical number. We seek a rule relating the size of the increment $n_{s}$ to the background level $n_{o}$ given a detection criterion of this sort.

First suppose that $n_{0}$ is large enough to permit normal.approximations. The probability of a false alarm is then fixed when

$$
\frac{c-n_{0}}{n_{0}^{1 / 2}}=k
$$

provided, of course, that $\mathrm{k}$ is constant. This critical number may be inserted into the analogous normal deviate constructed for the increment distribution,

$$
\frac{c \cdot\left(n_{s}+n_{0}\right)}{\left(n_{s}+n_{0}\right)^{1 / 2}}=0,
$$

where $n_{\mathrm{s}}$ is taken to be large enough to ensure that the increment is detectable with probability $1 / 2$. Accordingly:

$$
\begin{aligned}
n_{0}+k n_{o}^{1 / 2}-\left(n_{s}+n_{o}\right) & =0, \\
\frac{n_{s}}{n_{o}^{1 / 2}} & =k .
\end{aligned}
$$

This is de Vries' square root formula cited in the text. The constant $\mathrm{k}$ is determined by the false-alarm probability, and when the latter is fixed, a square root discrimination law emerges.

The expression in [A3] may be generalized by going to the square root transform:

$$
2\left(n_{\mathrm{s}}+\mathrm{n}_{\mathrm{o}}\right)^{1 / 2}-2\left(\mathrm{n}_{\mathrm{o}}\right)^{1 / 2} \cong \mathbf{d}^{\prime},
$$

where $d^{\prime}$ is the standard normal distance used to measure detectability in signal detection theory. The square root transform is an important normal approximation to the Poisson distribution. It is discussed in many sources (see, for example, Rao, 1952, pp. 209-210). It is also quite easy to prove the square root law in Equation [A3] via the square root transform (see McGill, 1966).

In any event, to obtain the pure-tone detection formulae used in this paper, we now assume the energy relation,

$$
\mathrm{n}=\mathrm{aE} \mathrm{E}^{1-\mathrm{c}} \text {. }
$$

Insert the latter into the square root transform Equation [A4] :

$$
\begin{gathered}
2 a^{1 / 2}\left[\left(E_{o}+E_{s}\right)^{(1-c) / 2}-E_{o}(1-c) / 2\right] \cong d^{\prime}, \\
2 a^{1 / 2} E_{o}(1-c) / 2\left[\left(1+\frac{E_{s}}{E_{o}}\right)^{(1-c) / 2}-1\right] \cong d^{\prime} .
\end{gathered}
$$

To reduce this further note that $(1-c) / 2$ is small and that

$$
\lim (1+x)^{m} \Rightarrow 1+m \log _{e}(1+x)
$$

Hence we write:

$$
2 a^{1 / 2} E_{\delta}^{(1-c) / 2}\left[\left(\frac{1-c}{2}\right) \quad \log _{e}\left(1+\frac{E_{s}}{E_{o}}\right)\right] \cong d^{\prime},
$$

and finally

$$
2(1-b) n_{o}^{1 / 2}, \log _{e}\left(1+E_{s} / E_{0}\right) \cong d^{\prime} \text {, }
$$

where $b=(1+c) / 2$. This is the form of the psychometric function studied in Equation 6 in the text of the paper.

To find our intensity discrimination law, we begin with Equation [A5] and proceed as follows:

$$
2 a^{1 / 2}(1-b) E_{o} 1-b\left(\log _{e}\left(1+E_{s} / E_{o}\right)\right] \cong d^{\prime},
$$

$$
\begin{array}{r}
2 a^{1 / 2}(1-b) E_{o}^{1-b}\left[\frac{E_{s}}{E_{0}}-\frac{1}{2}\left(\frac{E_{s}}{E_{o}}\right)^{2}+\frac{1}{3}\left(\frac{E_{s}}{E_{o}}\right)^{3} \ldots\right] \cong d^{\prime} \\
2 a^{1 / 2}(1-b) \frac{E_{s}}{E_{o}^{b}}\left[1-\frac{1}{2}\left(\frac{E_{s}}{E_{0}}\right)+\frac{1}{3}\left(\frac{E_{s}}{E_{o}}\right)^{2}\right. \\
\left.-\frac{1}{4}\left(\frac{E_{s}}{E_{0}}\right)^{3}\right] \ldots \cong d^{\prime} .
\end{array}
$$

The term in square brackets is very close to

$$
\begin{aligned}
& \left(1+\frac{E_{s}}{E_{0}}\right)^{-1 / 2}=1-\frac{1}{2}-\frac{E_{s}}{E_{0}}+\frac{3}{8}\left(\frac{E_{s}}{E_{0}}\right)^{2} \\
& -\frac{5}{16}\left(\frac{E_{s}}{E_{o}}\right)^{3}+\ldots
\end{aligned}
$$

Accordingly we have the following approximation for the detectability of pure tones.

$$
\frac{2 a^{3 / 2}(1-b) E_{s}}{\left(1+E_{s} / E_{o}\right)^{1 / 2} E_{o}^{b}}
$$

This is the intensity discrimination law given as Equation 3 in the text of the paper. 